\title{
El terremoto de 1647 de Chile central como un evento intraplaca: ¿otra amenaza para Chile metropolitano? ${ }^{1}$
}

\author{
Marco Cisternas ${ }^{2}$
}

\begin{abstract}
RESUMEN
El terremoto del 13 de mayo de 1647, la mayor catástrofe en la historia colonial de Chile central, es considerado como el segundo evento de la secuencia de terremotos interplaca que ha ocurrido con una sorprendente regularidad en esta parte de Chile. Sin embargo, el análisis histórico realizado sugiere que este terremoto, además de generar un enérgico y extenso sacudimiento en el valle central, no habría producido un tsunami. Ambas características podrían significar que se trató de un evento tipo intraplaca, originado superficialmente en la placa continental o profundo en la de Nazca. Los efectos reportados se asemejan más a los del terremoto de Chillán de 1939, un evento intraplaca de profundidad intermedia que aunque no produjo un tsunami generó grandes intensidades en el valle central y una enorme cantidad de víctimas. Si el terremoto de 1647 fue intraplaca, se plantea un problemático escenario de riesgo para la región más poblada del país.
\end{abstract}

Palabras clave: Terremoto de 1647, terremoto intraplaca, sismología histórica, Chile central.

\begin{abstract}
The May 13, 1647 earthquake, the larger catastrophe in the colonial history of central Chile, is considered as the second event of the inter-plate earthquakes series that has occurred at regular intervals in this part of Chile. However, this historical analysis suggests this earthquake, besides generating a strong and widespread shaking in the central valley, did not produced a tsunami. Both features could imply it was an intra-plate event, sourced in the surface of the continental plate or deeper in the Nazca plate. The reported effects resemble those from the 1939 ChiIlan earthquake, a middle-depth intra-plate event that although it did not produce a tsunami it does generated large intensities in the central valley and a huge number of victims. If the 1647 earthquake was intra-plate, a problematic risk scenario is set for the most populated region of the country.
\end{abstract}

Key words: 1647 earthquake, intra-plate earthquake, historical seismology, central Chile.

1 Se agradece el apoyo historiográfico de Fernando Torrejón y Nicolás Gorigoitía. Sin su colaboración esta publicación no hubiese sido posible. La investigación fue financiada por el Proyecto Fondecyt $\mathrm{N}^{\circ}$ 1110848. Artículo recibido el 28 de agosto de 2012 , aceptado el 15 de septiembre de 2012 y corregido el 30 de septiembre de 2012.

2 Escuela de Ciencias del Mar, Pontificia Universidad Católica de Valparaíso (Chile).

E-mail: marco.cisternas@ucv.cl 
Los grandes terremotos de Chile central $\left(32^{\circ}-35^{\circ} \mathrm{S}\right)$ parecen haber ocurrido a intervalos sorprendentemente regulares desde la llegada, y establecimiento permanente, de los españoles en 1541. Los eventos que han sido asignados a esta secuencia ocurrieron en 1575, 1647, 1730, 1822, 1906 y 1985, generando un intervalo promedio de $\sim 80$ años (Lomnitz, 1970; Kelleher, 1972; Comte et al., 1986; Beck et al., 1998; Barrientos, 2007). Esta regularidad se explicaría simplísticamente aplicando la teoría de la deformación elástica de las placas (Reid, 1910) a la zona de subducción de Chile central (Figura 1). Aquí, la subductante placa de Nazca y la cabalgante Sudamericana se enfrentan una contra la otra, convergiendo a una tasa de 6.6 cm/año (Angermann et al., 1999; Kendrick et al., 2003). La zona en que se encuentran ambas placas (interplaca) está trabada por lo que progresivamente se acumula tensión ( $\mathrm{A}$ en Figura $\left.N^{\circ} 1\right)$. Finalmente, cuando la tensión supera la fuerza del trabamiento, las placas se liberan abruptamente. De este modo, al considerar constante la tasa de convergencia en Chile central, se explicaría que después de 80 años de acumulación de convergencia las placas se liberan generando un gran terremoto a intervalos regulares.

En Chile central este escenario parece tan evidente que incluso se llegó a pronosticar acertadamente el terremoto de 1985 (Nishenko, 1985). Así, Chile metropolitano y en especial sus grandes centros urbanos, incluyendo los costeros de Valparaíso y San Antonio, podrían esperar tranquilamente que el próximo gran terremoto de la serie ocurra recién hacia la segunda mitad de este siglo, aproximadamente en el año 2065. Sin embargo, este auspicioso pronóstico tiene dos grandes debilidades. Primero, asume que el registro histórico está completo y que ningún terremoto se les ha escapado a los historiadores y sismólogos, puesto que si fuese de este modo, la supuesta regularidad ya no existiría. Recientemente se ha demostrado que aun es posible encontrar registros escritos de "nuevos" terremotos, no incluidos en los catálogos sísmicos. Cisternas et al. (2012) hallaron en España una carta que reporta la ocurrencia de un terremoto destructivo en Santiago en 1580, solo cinco años después del evento de 1575, que era considerado hasta ahora como el único terremoto del primer siglo de historia escrita de Chile central. Una segunda debilidad para realizar un pronóstico adecuado es asumir que todos los terremotos de la secuencia se originaron en la zona de interplaca. Para los terremotos preinstrumentales esta suposición es muy difícil de probar debido a que Chile central tiene cuatro grandes fuentes de terremotos (Figura $N^{\circ} 1$ ): está la ya mencionada mega falla de interplaca, en el límite de las placas de Nazca y Sudamericana, que produce los terremotos más grandes y frecuentes (A); fallas al interior de la placa de Nazca (intraplaca) debajo del mar y al oeste de la fosa oceánica, también llamadas out-rise (B); fallas al interior de la subductada placa de Nazca (intraplaca), conocidas como de "profundidad intermedia" (C); fallas superficiales en la placa continental (intraplaca), asociadas a los Andes (D) (Campos et al., 2005; Barrientos, 2007).

A diferencia de los eventos intraplaca (B, C, D), los terremotos interplaca (A) exhiben características evidentes que los permiten identificar como tales en el registro histórico. Cuando se libera la placa Sudamericana, una buena parte de la ruptura se produce bajo la costa, generando allí una mayor intensidad sísmica en comparación con el valle central. Adicionalmente, el destrabamiento de la placa continental produce notorios cambios en el nivel de la costa, tal y como ocurrió durante el terremoto de 2010 (Farías et al., 2010; Melnick et al., 2012), cuando algunos sectores se levantaron hasta 1,8 m. Ese mismo levantamiento del fondo del mar (i.e. plataforma y talud continental) golpea la columna de agua generando tsunamis que son fácilmente observables, como también ocurrió en el 2010. De este modo, cuando la descripción histórica de un terremoto pasado reporta gran intensidad en la costa, levantamiento o hundimiento costero y la generación de un tsunami, es posible afirmar que el sismo tuvo su origen en la zona de interplaca (A).

Usando fuentes históricas ya conocidas como otras recientemente encontradas en archivos chilenos, peruanos y españoles, el presente trabajo analiza las características del terremoto del 13 de mayo de 1647; sin duda, la mayor catástrofe de la historia colonial de Chile central. Se propone que este terremoto no tuvo las características de un evento interplaca. De ser esto correcto y considerando 
Figura $\mathrm{N}^{\circ} 1$

Zona de subducción y principales fuentes sísmicas en Chile central. La placa de Nazca desciende bajo la placa Sudamericana. La zona de contacto entre ambas (interplaca) se encuentra trabada

(A), acumulándose tensión progresivamente. Cuando se liberan se produce un terremoto interplaca en A. Las tres fuentes restantes están asociadas a fallas intraplaca. Fallas out-rise (B) y fallas de profundidad intermedia (C), en la placa de Nazca. Fallas superficiales asociadas a los Andes (D), en la placa Sudamericana.

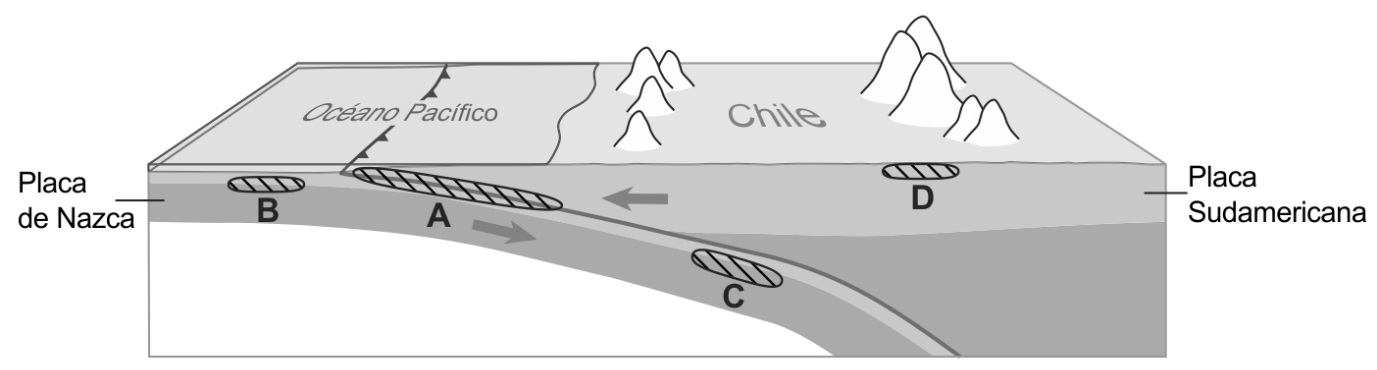

Fuente: Elaboración propia en base a Campos et al. (2005) y Atwater et al. (2005).

que los terremotos intraplaca son eventos extremadamente destructivos y de los que no se conoce su recurrencia, se concluye que Chile metropolitano enfrenta un segundo tipo de amenaza sísmica, quizás mucho más peligrosa para las ciudades del valle central, incluyendo especialmente a la sobrepoblada capital del país.

\section{Análisis del terremoto de Chile central de 1647}

De acuerdo a las crónicas, el terremoto del 13 de mayo de 1647 comenzó súbitamente a las, aproximadamente, 22:30 horas, generando daño a lo largo de $400 \mathrm{~km}$, entre Illapel (aprox. $31.5^{\circ} \mathrm{S}$ ) y Talca (aprox. 35.3 S). Devastó Santiago y mató alrededor de mil personas, es decir, a un quinto de su población (Montessus de Ballore, 1912: 18). El inusual, repentino y enérgico movimiento destruyó la mayoría de los edificios de la ciudad, tan pronto comenzó. El padre Juan González Chaparro, un estudioso jesuita chileno establecido en Lima, le escribe a su cofrade y compatriota, el padre Alonso de Ovalle, quien estaba en Roma, una carta fechada el 13 de julio de 1647, describiendo la reciente catástrofe en la patria común (Figura $N^{\circ} 2$ ):
“[...] súbito vino un temblor y terremoto tan horrible y espantoso que en menos de cuatro credos asoló y derribó toda la suntuosa pompa de los edificios de esta triste y afligida ciudad [...]. En tan repentino suceso, que sobrevino sin rumor ni ruido antecedente, como suele en otros temblores [...]".

El enérgico sacudimiento generó deslizamientos de rocas desde el cerro Santa Lucía (Montessus de Ballore, 1912: 19), un resultado que no se volvería a repetir en los subsecuentes terremotos de Chile central. A pesar de esta gran intensidad, el terremoto de 1647 no habría generado un tsunami en Valparaíso (Vicuña Mackenna, 1936: 601), que por ese entonces era una villa con dieciséis casas y dos iglesias, según una representación contemporánea (Ovalle, 1646).

Aunque Montessus de Ballore coincide con Vicuña Mackenna, respecto a la ausencia de un tsunami, diciendo que su foco "No fue submarino, pues no hubo movimiento alguno del mar" (Montessus de Ballore, 1912: 23), tres indicios, todos provenientes desde un único documento escrito en Santiago un año después del terremoto, les han sugerido a los sismólogos que sí hubo un tsunami en 1647. 
Figura $\mathrm{N}^{\circ} 2$

Extracto de la carta enviada desde Lima por el padre Juan González Chaparro al padre Alonso de Ovalle, quien estaba en Roma. Además de describir la catástrofe ocurrida en la patria común, entrega detalles de lo ocurrido en las costas de Perú.

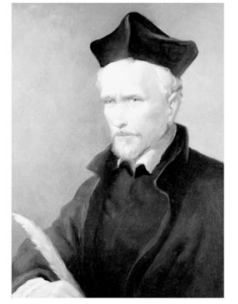

El Padre Alonso de Ovalle, afamado estudioso jesuita (izquierda), recibe en Roma una carta escrita en Lima por su cofrade Juan González por su cofrade Juan González
Chaparro. La carta vívidamente describe la catástrofe de 1647 (abajo). Aunque la información relativa a Santiago se basa en una carta de la Real Audiencia (Figura N ${ }^{\circ} 3$ ), González entrega importantes pistas de lo ocurrido en Perú, permitiendo clarificar ocurrido en Perú, permitiendo clarificar dado por la Audiencia.

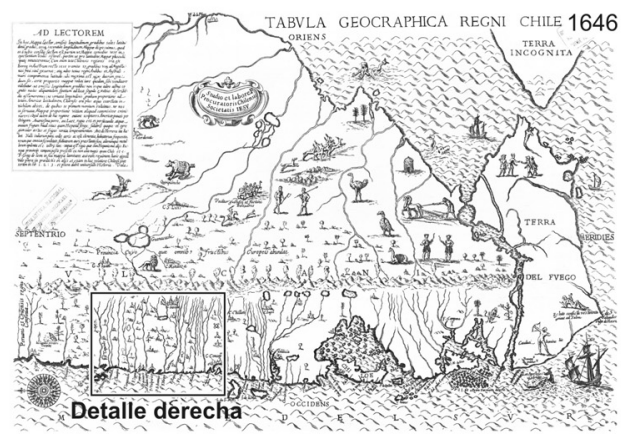

Tabula Geographica Regni Chile. Tabla Geográfica del Reino de Chile es una de las más antiguas representaciones del cono Suna un a 1647, sirvió de mapa de referencia torica del Reino de Chile escrita por el Padre Ovalle Se representan las principales poblaciones de Chile central para ese entonces (detalle a la derecha): Santiago (A), Concepción (B), Valparaíso (C) y Coquimbo, el puerto de La Serena (D)
A 13 de Mayo deste año de 47, a las 10 y media de la noche [...], súbito vino un temblor y terremoto tan horrible y espantoso que en menos de cuatro credos asoló y derribó [...] los edificios de esta triste y afligida ciudad, [...]. En tan repentino suceso, que sobrevino sin rumor ni ruido antecedente, como suele en otros temblores, [...] perecieron más de mil personas [...] los peñascos que están sobre el cerro Santa Lucia, caballero y contiguo a la ciudad, [...] se desgajaron dos de formidable grandeza [...] después de la primera conmoción que llevó tras si toda la ciudad, se repitieron dos temblores mayores que el primero, y no hallando en qué hacer presa, descuadernando la tierra la abrieron muchas bocas y grutas, arrojando tanta copia de agua espesa y turbia, que inundó las campiñas [...] y poco antes [del terremoto] se levantaron en esta costa del Perú horribles tormentas en las orillas del mar, tragando navios. Armose también el aire, congelando densas nubes y arrojando poco después granizo y recios aguaceros. [...] en el puerto de Arica había naufragado seis dia antes del terremoto el navio San Nicolás con 200,000 pesos [...]

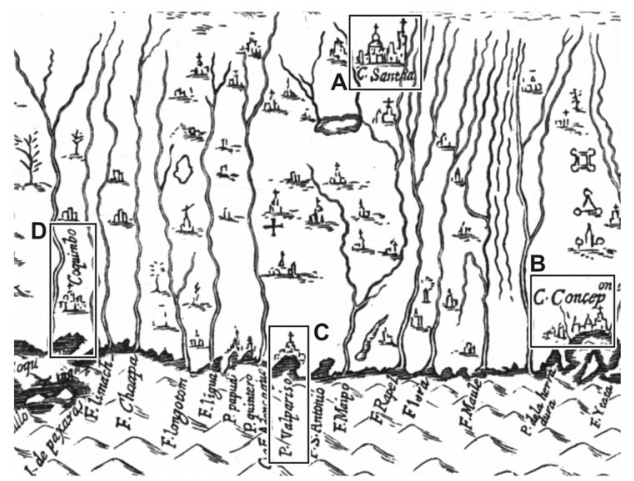

Fuente: Elaboración propia en base a carta del padre Juan González Chaparro, de la Compañía de Jesús, y de la Vice Provincia de Chile, para el padre Alonso de Ovalle [...]. Fondo histórico y bibliográfico José Toribio Medina, 1963, I, p. 475-484. Mapa obtenido en Ovalle (1646).

Tal sugerencia ha permitido que el terremoto de 1647 sea considerado dentro de la regular secuencia de terremotos interplaca de Chile central.

El documento en cuestión (Figura $\mathrm{N}^{\circ} 3$ ) fue enviado por la Real Audiencia, el mayor poder de justicia de Chile colonial, al rey de España el 12 de julio de 1648. La Audiencia reporta la ocurrencia de inusuales movimientos del mar:

i) "El mesmo dia [13 de Mayo 1647] salio la mar tan furiossamente contra la muralla de la cerca del puerto del Callao y con ser tan murada y fuerte se llevo un lienzo deIla". ii) "Y a siete de Mayo en el puerto de Arica sin biento se levanto el mar desusadamente [e] hizo varrar a un navio Sant Nicolas $[\ldots]^{\prime \prime}$.

iii) "Y por todos los puertos de esta costa advertieron los pescadores tanta inquietud y tanta extraordinaria violencia en las olas del mar que se subian sobre las cumbres mas altas $[\ldots]^{\prime \prime}$.

Aunque estas afirmaciones parecen confirmar la ocurrencia de un tsunami en 1647, hay algunas ambigüedades para interpretarlas como el resultado de un tsunami en Chile central. En primer lugar, debido a que el terremoto de 1647 ocurrió cerca de las 
22:30 horas en Chile central es difícil que el tsunami haya Ilegado "El mesmo dia" al Callao, que se encuentra a $2.400 \mathrm{~km}$ al norte de Valparaíso. El reciente tsunami de 2010, gatillado por una ruptura que alcanzó por su límite norte hasta $65 \mathrm{~km}$ al sur de Valparaíso, demoró 4 horas en Ilegar al Callao.

En segundo lugar, el referido naufragio del navío San Nicolás ocurrió, como la misma Audiencia lo afirma, el "siete de Mayo", es decir, seis días antes del terremoto. La fecha del naufragio es también corroborada en la carta remitida desde Lima por el padre Juan González Chaparro (Figura $N^{\circ} 2$ ):

"[...] en el puerto de Arica había naufragado seis día antes del terremoto el navío San Nicolás con 200,000 pesos".

Finalmente, cuando la audiencia reporta "Y por todos los puertos de esta costa", no precisa los lugares donde las olas fueron observadas. Se puede inferir, por coherencia en la redacción, que se refiere a las costa sur del Perú debido a que usa "esta costa", después de describir el naufragio de Arica.

Es muy probable que malas condiciones meteorológicas invernales en la costa sur del Perú expliquen la solitaria descripción de la Audiencia acerca de un tsunami en 1647. El padre Juan González Chaparro reporta (Figura $\left.\mathrm{N}^{\circ} 2\right)$ :

“y poco antes [del terremoto] se levantaron en esta costa del Perú horribles tormentas en las orillas del mar, tragando navíos. Armose también el aire, congelando densas nubes y arrojando poco después granizo y recios aguaceros $[\ldots]^{\prime \prime}$.

Debido a las pobres comunicaciones entre Perú y Chile por ese entonces, un viaje en barco entre Valparaíso y El Callao tomaba más de quince días en un solo sentido, es probable que las nuevas acerca de la tormenta peruana, que precedió al terremoto, Ilega-

Figura $N^{\circ} 3$

Reporte de la Real Audiencia al rey Felipe IV describiendo los efectos del terremoto de 1647. Las referencias a movimientos en el mar han servido de base para afirmar que este terremoto generó un tsunami.
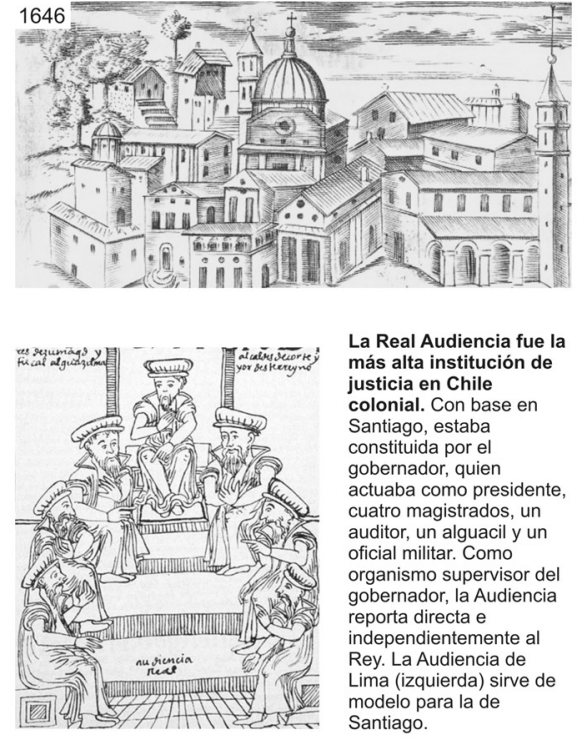

Imagen idealizada de Santiago impresa en Roma un año antes del terremoto de 1647 (izquierda). La capital tenía cerca de 5.000 habitantes hacia mediados del s. XVII. Una de cada cinco personas murió debido al repentino y enérgico sacudimiento. La Real Audiencia informa un año después al Rey Felipe IV los efectos del sismo (extractos abajo). Aunque se mencionan movimientos en el mar, hay algunas ambigüedades para interpretarlos como resultado de un tsunami en Chile central.

Escrivimos a V. M. el año passado de [1647] acavada de suceder la ruyna que padecio esta ciudad con el terremoto que le sobrevino a trece de mayo en la noche [...] con muerte de mill personas [...] se an ydo repitiendo mas de trecientos temblores pequeños [. ] Certifican los de Cuyo, de otra juzgaron que sedavan la vatalla unos montes con della que juzgaron que se davan la vatalla unos montes con otros [...], este no se oyo en Santiago por que cayo tan aplomo la ciudad [...]. El mesmo dia salio la mar tan furiossamente contra la muralla de la cerca del puerto del Callao y con ser tan murada y fuerte se llevo un lienzo della. $Y$ a siete de Mayo en el puerto de Arica sin biento se levanto el mar desusadamente se hizo pedazos contra unas a un navio Sant Nicolas [...] se hizo pedazos contra unas penas donde con muerte de catorce personas se perdieron mas de 200,000 p. Y por todos los puertos de esta costa advertieron los pescadores tanta inquietud y tanta extraordinaria violencia en las olas del mar que se subian sobre las cumbres mas altas [...] Aborto la tierra por los esteros y abras y cavidades hondas, radales tan furiosos de agua tan turbia [.. ]. Despidieron los radales tan furiosos de agua tan turbia [.... . Despidieron los montes peñascos de tal tamaño [...] que [...] pueden servir
de cerros no pequeños donde pararon. [...].

Fuente: Elaboración propia en base a Carta de la Real Audiencia de Chile sobre el terremoto del 13 de mayo de 1647, fechada el 12 de julio de 1648 en Santiago. Incluida en Gay (1852). Imágenes obtenidas en Ovalle (1646) arriba y Guamán Poma de Ayala (1613) abajo. 
ron atrasadas al devastado Santiago y fueron posteriormente interpretadas por la Audiencia como un hecho causa-efecto.

Quizás aun más revelador es la falta de un reporte de tsunami en la bahía de Concepción, una ensenada extremadamente propensa a amplificar tsunamis. Al igual que Valparaíso, la ciudad de Concepción, localizada en ese entonces en la cabecera de la somera bahía de Concepción, también parece carecer de un tsunami en 1647. Esto a pesar que los subsecuentes terremotos generados en Chile central (a la latitud de Valparaíso) produjeron olas que devastaron Concepción en 1730, inundaron Penco en 1906 y tuvieron amplitudes medidas de $1,8 \mathrm{~m}$ en Talcahuano en 1985. La bahía también ha amplificado tsunamis provenientes desde el extremo norte de Chile. El destacado hidrógrafo Francisco Vidal Gormaz, fundador del SHOA, reporta lo observado en la bahía de Concepción para el terremoto y tsunami de Arica de 1868 (Vidal Gormaz, 1880: 143):

"Con el terremoto de Arequipa de 1868 se produjo en Talcahuano una ola desbordante que comenzó por un retroceso del mar que hizo varar a los buques surtos en el puerto sobre 7 i 7.5 metros de agua, produciéndose en seguida una ola que inundó parte del pueblo y que subió no menos de 4.5 metros sobre el nivel ordinario de marea Ilena, ocasionando grandes perjuicios en el pueblo".

Esta descripción no deja de ser trascendente para la evaluación del riesgo de la actual ciudad de Talcahuano, toda vez que se está a la espera de que ocurra un nuevo gran terremoto en el norte de Chile (Comte \& Pardo, 1991).

El análisis de los registros del mareógrafo de Talcahuano demuestra que la bahía de Concepción incluso ha amplificado tsunamis de campo muy lejano. Es el caso del tsunami generado por el terremoto de Alaska de 1964, que produjo amplitudes de hasta $3 \mathrm{~m}$ en Talcahuano. El fenómeno se repitió recientemente con el tsunami de Japón de 2011, cuando amplitudes de $4 \mathrm{~m}$ fueron registradas por el mismo instrumento. Adicionalmente a todo lo anterior, la bahía de Concepción ha sido afectada por sus propios tsunamis, originados en la interplaca a la latitud de Concepción, que ocurrieron en 1570, 1657, 1751, 1835 y 2010. Esta propensión a sufrir tsunamis destructivos forzó a los vecinos de Concepción a trasladar la ciudad tierra adentro después el tsunami de 1751, dejando atrás al poblado de Penco.

Aunque Concepción tenía hacia 1647 una bien establecida población, que habitaba más de 100 casas que bordeaban la playa (Campos-Harriet, 1989), el reporte del gobernador de Chile, Martín de Mujica, en Concepción durante el terremoto, no menciona movimientos del mar después de sentir el distante sacudimiento que destruía su ciudad capital. De Mujica lacónicamente reporta (Figura $N^{\circ}$ 4):

"A esta ciudad [Concepción] llego el temblor y fue á las diez y media de la noche y aun que duro buen tiempo no hiço daño alguno".

Aunque este reporte implica evidencia negativa, la falta de mención a anomalías en el mar es a lo menos sorprendente, especialmente si se considera que el emplazamiento de Concepción estaba casi al nivel del mar. Un documento anónimo, escrito ochenta y tres años más tarde, con ocasión del posterior tsunami de 1730, confirma que los habitantes de la ciudad temían incluso por inundaciones de las mareas altas:

"Su situación es a orillas del mar, y aun mucha parte de ella sobre su misma playa, $[\ldots]$. Parte casi por medio a la Ciudad un Rio, mas rico por la calidad, que por el caudal de sus aguas [...]; pero como el plan, por donde corre sea igual a el del mar, suben por el las mareas, entrando mui adentro de la ciudad, y a veces por no caver en su cauce, se explayan por las calles con peligro de los edificios, y siempre con susto de que creciendo los anegue" ${ }^{\prime \prime}$.

\footnotetext{
3 Relación del lastimoso y horrible estrago de la Ciudad de la Concepción del Reyno de Chile, causado del temblor e inundación del Mar que anegó el día 8 de julio de 1730. Archivo Histórico Nacional, Colección Sergio Fernández Larraín, Vol. XXIII, pz. 5.
} 


\section{Figura $\mathrm{N}^{\circ} 4$}

Reporte del gobernador de Chile Martín de Mujica al Rey describiendo los efectos del terremoto de 1647 en Santiago y Concepción. A pesar de resultar devastador en Santiago, no "hizo daño alguno" en Concepción.
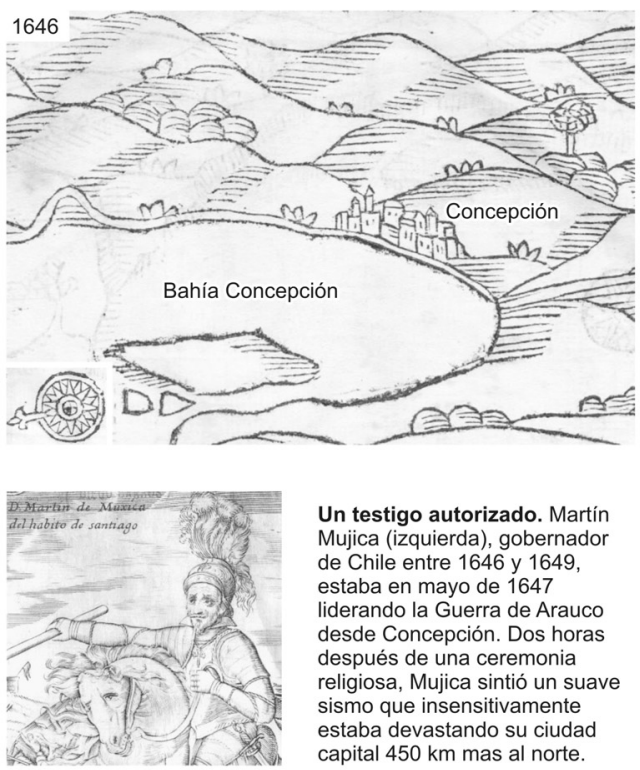

Un testigo autorizado. Martín Mujica (izquierda), gobernador de Chile entre 1646 y 1649 , de Chile entre 1646 y 1649 liderando la Guerra de Arauco desde Concepción. Dos horas después de una ceremonia sismo que insensitivamente (a) religiosa, Mujica sintió un suave capital $450 \mathrm{~km}$ mas al norte.
Una ciudad muy propensa a tsunamis. La antigua Concepción, fundada en la cabecera de la bahía de Concepción, una ensenada somera y expuesta al norte, fue establecida sobre una estrecha planicie costera (izquierda). Tanto Concepción como su poblado descendiente Penco fueron destruidas o dañadas por tsunamis generados a su misma latitud en $1570,1657,1751$ tsunamis generados a su misma latitud en $1570,1657,1751$,
1835 y 2010 . Adicionalmente, tsunamis generados en Chile 1835 y 2010 . Adicionalmente, tsunamis generados en Chile
central, a la latitud de Valparaiso, también se han amplificado en la bahía, destruyendo Concepción en 1730, inundando Penco en 1906 y alcanzando $2 \mathrm{~m}$ de amplitud en Talcahuano en 1985. Sin embargo, sorprendentemente, el gobernador de Chile Martín Mujica, en Concepción durante el terremoto de Chile central de 1647, no reporta daños o movimientos del mar después del sismo.

Señor - Cerrando ya el despacho que en esta ocasion hago á V.M. [Vuestra Majestad] Recivi la carta inclusa que la Audiencia de la Ciudad de Santiago me escrivio dando á viso de la lamentable Ruina que un gran terremoto hiço en toda aquella ciudad; Bien se deja ber por ella quan del todo quiso Dios nuestro señor asolarla [...], no queda piedra sobre piedra [...]; Las muertes fueron tantas que perecieron familias enteras; no se ha visto en todas las indias tan unibersal ruina [...]. A esta ciudad [Concepción] llego el temblor y fue á las diez y media de la noche y aun que duro buen tiempo no hiço daño alquno, atribuyolo dignamente á que havia dos oras, que el Reverendo obispo desta ciudad, y yo con la mayor parte del pueblo haviamos ydo adar gracias, á nuestra señora de las nieves [...] Concepcion de Chile Mayo veintiocho de mil seiscientos cuarenta y siete - D. Martin de Muxxica.

Fuente: Elaboración propia en base a carta del gobernador de Chile Martín de Mujica a S.M., fechada el 28 de mayo de 1647 en Concepción. Archivo José Toribio Medina, Sala Medina, Carta № 2578. Foja 296, Tomo 139. Imagen obtenida en Ovalle (1646).

De esta forma es difícil, en caso de haber ocurrido un tsunami en 1647, que el gobernador no reportara alguna secuela en Concepción. Considerando lo anterior, se infiere que el terremoto de 1647, además de producir un devastador y repentino sacudimiento en la depresión intermedia de Chile central, no produjo un tsunami, al menos en la costa central y centro sur de Chile.

\section{La catástrofe de 1647 como un terremoto intraplaca}

El análisis de las fuentes históricas sugiere que el terremoto de 1647 produjo un inusual, repentino, y enérgico sacudimiento en el valle central y que además carece de un tsunami en las costas del centro y centro sur de Chile. Esta combinación podría significar que el terremoto de 1647 no fue generado en la interplaca, en la zona de contacto entre las placas de Nazca y Sudamericana (A), sino que al interior de alguna de ellas.
Si se descarta la fuente interplaca para el evento de 1647, quedan como candidatos posibles las fallas out-rise en la placa de Nazca (B), las fallas de profundidad intermedia en la placa subductada (C) y las fallas corticales asociadas a los Andes (D). De las tres, la fuente menos probable son las fallas out-rise, pues por su localización, en la placa de Nazca y más allá de la fosa oceánica, sus efectos en el continente debiesen ser menores. Así al menos ocurrió con los terremotos out-rise de Chile central de 1971 (M 5.8), 1973 (M 5.9), 1981 (M 7.2), 1982 (M 5.2), 1995 (M 5.6) y 2001 (M 7); Clouard et al., 2007, que no causaron daños en la costa y apenas fueron percibidos en el valle central. A pesar de lo anterior, los terremotos out-rise sí pueden generar tsunamis, como ocurrió durante el desastroso tsunami del noreste de Japón de 1896 (Satake \& Tanioka, 1999) y el tsunami de Samoa de 2009 (Beavan et al., 2010).

De este modo, solo quedan dos probables fuentes para el terremoto de 1647, una 
falla cortical asociada a los Andes o una falla de profundidad intermedia en la subductada placa de Nazca. La sugerencia de una fuente intraplaca cortical para el evento de 1647 no es nueva. Lomnitz (1970) propone una falla asociada a la Cordillera de la costa, estimando un epicentro a $80 \mathrm{~km}$ de Santiago. Posteriormente, el mismo Lomnitz (1983) revisita los principales argumentos dados previamente y propone un epicentro en la costa o mar adentro, sugiriendo una fuente en la interplaca. Finalmente, basado en un reconocimiento geológico, Lomnitz (2004) especula que la fuente del terremoto de 1647 podría haber estado asociada a la falla San Ramón, una falla inversa que corre a lo largo del piedemont andino al este de Santiago (Farías et al. 2010a, Armijo et al., 2010). Aunque el registro instrumental no ha detectado sismicidad cortical asociada a la falla San Ramón durante los últimos 30 años (Barrientos, 2007), recientemente se ha reportado el hallazgo de un escarpe de falla que muestra actividad entre los últimos 22.000 y 8.000 años (Vargas y Rebolledo, 2012). En base a estas observaciones, los autores proponen que la falla San Ramón podría generar terremotos con una magnitud estimada entre M 6.9 y 7.4. Otras fallas corticales, que también podrían presentar una amenaza para las ciudades localizadas en la depresión intermedia de Chile central, han sido reconocidas en levantamientos geológicos recientes (Charrier et al., 2005; 2007). Entre ellas destaca una posible extensión hacia el norte de la falla EI Fierro (Comte et al., 2008; Pardo et al., 2008; Farías et al., 2010b). Una expresión de este tipo de sismicidad cortical andina se observó durante el terremoto de Las Melosas en 1958, cuyo epicentro estuvo solo a $60 \mathrm{~km}$ al sureste de Santiago (Barrientos, 2007). Aunque el sismo alcanzó una magnitud 7 , se reportaron derrumbes y caminos cortados cerca del epicentro. Sin embargo, en esa ocasión, Santiago no sufrió daños de consideración (Lomnitz, 1961).

Finalmente, la posibilidad de una fuente al interior de la placa de Nazca, a profundidades intermedias, pareciera ser el peor escenario posible para la capital del país. Este tipo de terremotos pueden ser extremadamente destructivos debido a que tienen altas caídas del estrés acumulado (Kausel, 1991). Adicionalmente, comparados con los interplaca, liberan una mayor proporción de energía en alta frecuencia, lo que genera un mayor grado de destrucción en edificaciones bajas (e.g. Singh et al., 2000). Así, los terremotos de profundidad intermedia generan proporcionalmente mucho mayor daño en las proximidades del epicentro en comparación con eventos interplaca de similar magnitud (Astroza et al., 2005). Todas estas características sumadas a la localización de sus hipocentros, justo bajo el valle central, hacen que los terremotos intraplaca de profundidad intermedia puedan ser extremadamente destructivos en la depresión intermedia de Chile central. El terremoto de Chillán del 24 de enero de 1939 (M 7.8) es el más catastrófico ejemplo conocido de este tipo de sismos. Con una fuente intraplaca, al interior de la placa de Nazca y a una profundidad intermedia (Campos \& Kausel, 1990, Beck et al., 1998; Campos et al., 2002), produjo aproximadamente 30.000 víctimas fatales, más que cualquier otro terremoto en toda la historia de Chile (Lomnitz, 1970).

Considerando que los terremotos corticales, asociados a los Andes, presentan una importante tasa de atenuación de intensidad (Campos et al., 2005), y que sus expresiones superficiales de fallamiento se extienden solo por unas decenas de kilómetros (Charrier et al., 2005; 2007; Farías et al. 2010a; Armijo et al., 2010), es difícil sostener que puedan generar daños que se extiendan latitudinalmente a escala de centenares de kilómetros, como los producidos por el evento de 1647. En esa ocasión se reportaron daños a lo largo de $400 \mathrm{~km}$, entre los ríos Choapa y Maule (Montessus de Ballore, 1912). El caso más representativo de terremoto cortical de Chile central, Las Melosas en 1958 (M 6.9), generó efectos evidentes en un radio muy limitado alrededor de su zona epicentral (Lomnitz, 1961). En cambio, el terremoto de Chillán produjo daños a lo largo de $230 \mathrm{~km}$, entre Linares $\left(35,8^{\circ}\right)$ y Los Angeles $\left(37,5^{\circ}\right.$; Lomnitz, 1970). De este modo, es probable que el terremoto de 1647 haya tenido su fuente en la misma zona sismogénica que la tuvo el terremoto de Chillán de 1939. Si el terremoto de 1647 ocurrió dentro de la subductada placa de Nazca, como lo hizo el terremoto de ChiIlán, esto plantearía un problemático escenario de peligro para la región metropolitana, la más densamente poblada del país. 


\section{Consideraciones finales}

El terremoto de 1647 , sin duda la mayor catástrofe sísmica de Chile colonial, tuvo una inusual intensidad en la depresión intermedia de Chile central y carece de un tsunami reportado en la costa centro y centro sur de Chile. Ambas características sugieren que este terremoto no fue generado en la zona de interplaca, entre la placa de Nazca y Sudamericana, sino al interior de alguna de ellas.

Considerando las zonas sismogénicas de Chile central, es posible que el terremoto de 1647 haya sido generado al interior de la placa Sudamericana en una falla superficial asociada a los Andes o, alternativamente, al interior de la subductada placa de Nazca a una profundidad intermedia.

Debido a que los terremotos superficiales presentan una tasa de atenuación alta y que las expresiones superficiales de sus fallas solo tienen unas decenas de kilómetros, es difícil explicar el extenso daño latitudinal reportado en 1647 mediante este tipo de fuente. Los resultados reportados para el terremoto de 1647 se asemejan más a los generados por el terremoto de Chillán de 1939, que no produjo un tsunami, tuvo una alta intensidad en el valle central generando destrucción a lo largo de $200 \mathrm{~km}$ y produjo una enorme cantidad de víctimas.

Si el terremoto de 1647 ocurrió dentro de la subductante placa de Nazca, al igual que lo hizo el terremoto de Chillán de 1939, se plantea un problemático escenario de riesgo para la región que concentra a la mitad de la población del país.

\section{Referencias bibliográficas}

ANGERMANN, D.; KLOTZ, J. \& REIGBER, C. Space-geodetic estimation of the Nazca-South America Euler vector. Earth and Planetary Science Letters, 1999, № 171, p. 329-334.

ARMIJO, R.; RAULD, R.; THIELE, R.; VARGAS, G.; CAMPOS, J.; LACASSIN, R. \& KAUSEL, E. The West Andean Thrust, the San Ramón Fault, and the seismic hazard for San- tiago, Chile. Tectonics, 2010, № 29, TC2007, doi: 10.1029/2008TC002427.

ASTROZA, M.; SANDOVAL, M. y KAUSEL, E. Estudio comparativo de los efectos de los sismos chilenos de subducción del tipo intraplaca de profundidad intermedia. En: Congreso Chileno de Sismología e Ingeniería Antisísmica, Concepción, 2005.

ATWATER, B.; MUSUMI-ROKKAKU, S.; SATAKE, K.; TSUJI, Y.; UEDA, K. \& YAMAGU$\mathrm{CHI}, \mathrm{D}$. The orphan tsunami of 1700; Japanese clues to a parent earthquake in North America. Seattle: U.S. Geological Survey Professional Paper, 1707. University of Washington Press, 2005.

BARRIENTOS, S. Earthquakes in Chile. In: MORENO, T. \& GIBBONS, W. (Editors). Geology of Chile. London: The Geological Society, 2007, p. 263-287.

BEAVAN, J.; WANG, X.; HOLDEN, C.; WILSON, K.; POWER, W.; PRASETYA, G.; BEVIS, M. \& KAUTOKE, R. Near-simultaneous great earthquakes at Tongan megathrust and outer rise in September 2009. Nature, 2010, $N^{\circ} 466$, p. 959-963.

BECK, S.; BARRIENTOS, S.; KAUSEL, E., \& REYES, M. Source characteristics of historical earthquakes along central Chile subduction zone. Journal of South American Sciences, 1998, No 11, p. 115-129.

CAMPOS, J.; RUIZ, S.; PÉREZ, A.; RUIZ, J.; KAUSEL, E.; THIELE, R.; SARAGONI, R. y SEPÚLVEDA, S. Terremotos corticales de Las Melosas 1958, Chusmiza 2001 y Curicó 2004: un análisis comparativo de los terremotos de Northridge 1994 y Kobe 1995; nuevos antecedentes para el peligro sísmico en Chile. En: Congreso Chileno de Sismología e Ingeniería Antisísmica, Concepción, 2005.

CAMPOS, J. \& KAUSEL, E. The large 1939 intraplate earthquake of southern Chile. Seismological Research Letters, 1990, Nº61, p. 43.

CAMPOS, J.; HATZFELD, D.; MADARIAGA, R.; LÓPEZ, G.; KAUSEL, E.; ZOLLO, A.; IANNACONE, G.; FROMM, R.; BARRIENTOS, S. \& LYON-CAEN, H. A seismological study of the 1835 seismic gap in south-central 
Chile. Physics of the Earth and Planetary Interiors, 2002, N 132, p. 177-195.

CAMPOS-HARRIET, F. Historia de Concepción 1550-1988. Santiago de Chile: Editorial Universitaria, 1989.

CHARRIER, R.; BUSTAMANTE, M.; COMTE, D.; ELGUETA, S.; FLYNN, J.J.; ITURRA, N.; MUÑOZ, N.; PARDO, M.; THIELE, R. \& WYSS, A.R. The Abanico Extensional Basin: regional extension, chronology of tectonic inversion, and relation to shallow seismic activity and Andean uplift. Neues Jahrbuch für Geologie und Paläontologie, 2005, № 236, p. 43-47.

CHARRIER, R.; PINTO, L. \& RODRÍGUEZ, M.P. Tectonostratigraphic evolution of the Andean orogen in Chile. In: MORENO, T. \& GIBBONS, W. (Editors). Geology of Chile. London: The Geological Society, 2007, p. 21114.

CISTERNAS, M.; TORREJÓN, F. \& GORIGOITÍA, N. Amending and complicating Chile's seismic catalog with the Santiago earthquake of 7 August 1580. Journal of South American Sciences, 2012, №33, p. 102-109.

CLOUARD, V. ; CAMPOS, J. ; LEMOINE, A.; PÉREZ, A. \& KAUSEL, A. Outer rise stress changes related to the subduction of the Juan Fernandez Ridge, central Chile. Journal of Geophysical Research, 2007, № 112, B05305, doi:10.1029/2005JB003999.

COMTE, D.; EISEMBERG, A.; LORCA, E.; PARDO, M.; PONCE, L.; SARAGONI, R.; SINGH, S.K. \& SUÁREZ, G. The 1985 central Chile earthquake: a repeat of previous great earthquakes in the region? Science, 1986, $\mathrm{N}^{\circ}$ 233 , p. 449-453.

COMTE, D. \& PARDO, M. Reappraisal of great historical earthquakes in the northern Chile and southern Peru seismic gaps. Natural Hazards, 1991, N 4, p. 23-44.

COMTE, D.; FARÍAS, M.; CHARRIER, R. \& GONZÁLEZ, A. Active tectonics in the Central Chilean Andes: 3D tomography based on the aftershock sequence of the 28 August 2004 shallow crustal earthquake. In: 7th In- ternational Symposium on Andean Geodynamics, Nice, 2008.

FARÍAS, M.; VARGAS, G.; TASSARA, A.; CARRETIER, S.; BAIZE, S.; MELNICK, D. \& BATAILLE, K. Land-level changes produced by the $2010 \mathrm{Mw}$ 8.8 Chile earthquake. Science, 2010a, N³29, p. 916.

FARÍAS, M.; COMTE, D.; CHARRIER, R.; MARTINOD, J.; DAVID, C.; TASSARA, A.; TAPIA, F. \& FOCK, A. Crustal-scale structural architecture in central Chile based on seismicity and surface geology: Implications for Andean mountain building. Tectonics, 2010b, No29, TC3006, doi:10.1029/2009TC002480.

KAUSEL, E. The influence of large thrust and normal earthquakes in the assessment of the seismic hazard. In: Workshop New Horizons in Strong Ground Motion: Seismic Studies and Engineering Practice, Santiago de Chile, 1991.

KELLEHER, J. Rupture zones of large South American earthquakes and some predictions. Journal of Geophysical Research, 1972, N ${ }^{\circ}$ 77, p. 2087-2103.

KENDRICK, E.; BEVIS, M.; SMALLEY, R.; BROOKS, B.; VARGAS, R.; LAURIA, E. \& FORTES, L. The Nazca-South America Euler vector and its rate of change. Journal of South American Earth Sciences, 2003, No 16, p. 125-131.

LOMNITZ, C. Los terremotos del 4 de Septiembre de 1958 en el Cajón del Maipo. Anales de la Facultad de Ciencias Físicas y Matemáticas de la Universidad de Chile, 1961, Nº18, p. 279-306.

LOMNITZ, C. Major earthquakes and tsunamis in Chile during the period 1535 to 1955. Geologische Rundschau, 1970, № 59, p. 938-960.

LOMNITZ, C. On the epicenter of the great Santiago earthquake of 1647. Bulletin of the Seismological Society of America, 1983, $N^{\circ} 73$, p. 885-886.

LOMNITZ, C. Major earthquakes of Chile: a historical survey, 1535-1960. Seismological Research Letters, 2004, №75, p. 368-378. 
MELNICK, D.; CISTERNAS, M.; MORENO, M. \& NORAMBUENA, R. Estimating coseismic coastal uplift with an intertidal mussel: calibration for the 2010 Maule, Chile earthquake $(\mathrm{Mw}=8.8)$. Quaternary Science Reviews, 2012, N42, p. 29-42.

MONTESSUS DE BALLORE, F. Historia sísmica de los Andes Meridionales al sur del paralelo XVI. Santiago de Chile: Imprenta Cervantes, 1912.

NISHENKO, S.P. Seismic potential for large and great interplate earthquakes along the Chilean and Southern Peruvian margins of South America: a quantitative reappraisal. Journal of Geophysical Research, 1985, Nº 90, p. 3589-3615.

OVALLE, A. Historica relacion de Reyno de Chile. Roma: Impreso por Francisco CabaIlo, 1646 .

PARDO, M.; VERA, E.; MONFRET, T. \& YÁÑEZ, G. Crustal seismicity and 3D seismic wave velocity models in the Andes cordillera of Central Chile $\left(33^{\circ}-34.5^{\circ} \mathrm{S}\right)$ from local earthquakes. In: 7th International Symposium on Andean Geodynamics, Nice, 2008.

REID, H.F. The Mechanics of the Earthquake, The California Earthquake of April
18, 1906, Report of the State Investigation Commission. Washington, D.C: Carnegie Institution of Washington, 1910.

SATAKE, K. \& TANIOKA, Y. Sources of tsunami and tsunamigenic earthquakes in subduction zones. Pure and Applied Geophysics, $1999, N^{\circ} 154$, p. 467-483.

SINGH, S.K.; ORDAZ, M.; ALCÁNTARA, L.; SHAPIRO, N.; KOSTOGLODOV, V.; PACHECO, J.; ALCOCER, S.; GUTIÉRREZ, C.; QUAAS, R.; MIKUMO, T. \& OVANDO, E. The Oaxaca Earthquake of 30 September $1999(\mathrm{Mw}=7.5)$ : a normal-faulting event in the subducted Cocos plate. Seismological Research Letters, 2000, Nº71, p. 67-77.

VARGAS, G. \& REBOLLEDO, S. Paleosismología de la Falla San Ramón e implicancias. En: XIII Congreso Geológico de Chile, Antofagasta, 2012.

VICUÑA MACKENNA, B. Historia de Valparaíso. Santiago de Chile: Imprenta Dirección General de Prisiones, 1936.

VIDAL GORMAZ, F. Jeografía náutica de la República de Chile. Santiago de Chile: Imprenta Nacional, 1880. 
